\title{
Development and validation of an UHPLC-Orbitrap-HRMS method for rapid determination of endogenous L-carnitine in patients on hemodialysis/peritoneal dialysis and its application to promote rational drug use
}

\author{
Wen-Ting Jiang ${ }^{1 \#}$, Liu-Xing Tang ${ }^{1 \#}$, Jie Pan ${ }^{1}$, Cun-Jin Su ${ }^{1}$, Zhu Shen ${ }^{1}$, Zhan-Hong Hu ${ }^{1}$, Zhou-Bing Zhan ${ }^{2}$, \\ Ai-Ming Shi ${ }^{1}$ \\ ${ }^{1}$ Department of Pharmacy, The Second Affiliated Hospital of Soochow University, Suzhou, China; ${ }^{2}$ Department of Nephrology, The Second \\ Affiliated Hospital of Soochow University, Suzhou, China \\ Contributions: (I) Conception and design: AM Shi, CJ Su; (II) Administrative support: ZB Zhan, J Pan; (III) Provision of study materials or patients: \\ LX Tang; (IV) Collection and assembly of data: WT Jiang; (V) Data analysis and interpretation: Z Shen, ZH Hu; (VI) Manuscript writing: All \\ authors; (VII) Final approval of manuscript: All authors. \\ \#These authors contributed equally to this work. \\ Correspondence to: Ai-Ming Shi. Department of Pharmacy, The Second Affiliated Hospital of Soochow University, 1055 San Xiang Road, Suzhou \\ 215004, China. Email: sam740411@163.com; Zhou-Bing Zhan. Department of Nephrology, The Second Affiliated Hospital of Soochow University, \\ 1055 San Xiang Road, Suzhou 215004, China. Email: zhanzhoubing@foxmail.com.
}

Background: L-carnitine is an endogenous vitamin-like amino acid derivate which plays an essential role in energy metabolism and can be easily lost via dialysis. Deficiency of L-carnitine has great effects on many aspects of bodily functions. To determine the deficiency degree and adjust the supplementation dose, a rapid, sensitive, and specific method for the detection of endogenous L-carnitine in the plasma of dialysis patients using ultra-high performance liquid chromatography-Orbitrap high resolution mass spectrometry (UHPLCOrbitrap-HRMS) was developed and validated.

Methods: The plasma samples were processed by protein precipitation and centrifugation before analysis using UHPLC-Orbitrap-HRMS. Sample separation was achieved with a hydrophilic interaction liquid chromatography (HILIC) column, using an isocratic elution with a runtime of $5 \mathrm{~min}$. The separated analytes were detected by positive ionization mode in full scan mode and targeted-single ion monitoring (t-SIM) mode. Mildronate was used as the internal standard (IS).

Results: All the plasma could be detected in the range of 6.169 to $197.394 \mu \mathrm{M}$, with adequate accuracy, precision, and recovery. The method was validated in fortified validation with relative standard deviations (RSD) 5.15-8.74\%. This method was applied to the analysis of 105 dialysis patients and 39 healthy participants, the results revealed that peritoneal dialysis patients without L-carnitine supplementation should pay more attention to L-carnitine monitoring, meanwhile, all the hemodialysis patients were advised to be routinely given a full dose of L-carnitine, no matter whether they had taken L-carnitine or not.

Conclusions: This study developed a simple and rapid UHPLC-Orbitrap-HRMS method for detection of endogenous L-carnitine in dialysis patients, which could be useful to promote rational drug use.

Keywords: L-carnitine; dialysis; UHPLC-Orbitrap-HRMS; fortified validation; rational drug use

Submitted Nov 09, 2021. Accepted for publication Jan 19, 2022.

doi: 10.21037/atm-21-6784

View this article at: https://dx.doi.org/10.21037/atm-21-6784

^ ORCID: 0000-0002-4912-5168. 


\section{Introduction}

L-carnitine (Figure 1A) is an endogenous vitamin-like amino acid derivate, and it plays an essential role in oxidative metabolism and ketogenesis by decreasing the intramitochondrial acetyl-CoA/CoA ratio through the trapping of acetyl groups $(1,2)$. In addition, L-carnitine is a low molecular weight hydrophilic substance which can be excreted by kidneys. Kidney disease can disturb L-carnitine homeostasis, while L-carnitine can be easily lost via dialysis (3). It has been illustrated that about $95 \%$ of dialysis patients experience carnitine deficiency (4). Insufficient carnitine synthesis is another reason for the lack of L-carnitine in patients on hemodialysis and peritoneal dialysis. The effect of L-carnitine deficiency on patient health is profound, impacting on serum lipid, red blood cells, and cardiac and skeletal muscle (5). Because of the recent significant advances in dialysis therapy, the purpose of dialysis therapy is not only to prolong the patients' life, but also to restore the pre-dialysis state of health as much as possible. Furthermore, L-carnitine supplementation improves the complications of patients with kidney disease, such as erythropoietin-resistant anemia, cardiac symptoms, muscle weakness, impaired protein metabolism, and insulin resistance (6). Several recent studies have demonstrated that L-carnitine supplementation could suppresses inflammation by reducing levels of interleukin-6 (IL-6), C-reactive protein (CRP), tumor necrosis factor- $\alpha$ (TNF- $\alpha)(7,8)$. Besides, L-carnitine supplementation could attenuate oxidative stress responses by increasing superoxide dismutase (SOD) or glutathione $(7,9)$.

In order to provide personalized pharmacy services to dialysis patients, promote rational drug use, and reduce the waste of social resources and the economic burden of patients, we need to know the degree of individual L-carnitine deficiency. Currently, the common method for detecting carnitine utilizes solid-phase extraction column, derivatization, and high-performance liquid chromatography-tandem mass spectrometry (HPLC-MS) $(10,11)$. For the purpose of obtaining more reliable results, higher sample throughput, and simplifying the operation process, there is an increasing demand for the development of an ultra-high performance liquid chromatography (UHPLC) coupled to mass spectrometry (MS) analytical method (10,12-15).

However, owing to its design, it has a drawback that could be seriously affected by matrix effects. Matrix effects may reduce the sensitivity, precision, and accuracy of the method $(15,16)$. In addition, there is no target-free matrix for the detection of endogenous compounds, which leads to quantification difficulties. A number of approaches have been developed to solve the problem, including background subtraction $(17,18)$, standard addition (19), artificial matrix $(11,20)$, analyte-depleted biological matrix (21), and stableisotope labeled surrogate analytes $(22,23)$. Although these approaches have promoted the development of quantitative methodologies for endogenous substances, they still have various respective shortcomings, including limited sensitivity, the matrix effect, and recovery inconsistency of biological and the corresponding surrogate matrix (24).

Herein, we have described a simple, rapid, selective and highly sensitive ultra-high performance liquid chromatography tandem Orbitrap high resolution mass spectrometry (UHPLC-Orbitrap-HRMS) method for the determination of L-carnitine in human plasma using a hydrophilic interaction liquid chromatography (HILIC) column. Its method run time is $5 \mathrm{~min}$, and $50 \mu \mathrm{L}$ plasma is needed for analysis. In this study, mildronate (MILD; Figure $1 B$ ) was used as the internal standard (IS), because of its similarity in structure, retention time, and ionization to the molecular target (25). UHPLC offers the possibility of rapid and efficient sample separation. Modern mass spectrometry is usually equipped with mass analyzer with higher resolution and accuracy, such as time of flight (TOF) and Orbitrap (26). Most of the interfering ions are effectively eliminated by the narrow window of mass defect filtering (MDF) template, which enable the researchers to accurately capture the target molecular (27). The assay developed here incorporated matrix effects from different sources into quantitation for the first time. In addition, fortified validation was emphasized to ensure the reliability and accuracy of this assay. It is worth mentioning that there is no need to prepare analyte-depleted biological matrix or artificial matrix, which simplifies the analysis procedure and guaranteed the authenticity of the results. Plasma concentrations of L-carnitine were accurately measured using one calibration curve constructed with anyone's untreated plasma.

We present the following article in accordance with the MDAR reporting checklist (available at https://atm. amegroups.com/article/view/10.21037/atm-21-6784/rc).

\section{Methods}

\section{Study participants and sample collection}

This study was approved by the Ethics Committee of The Second Affiliated Hospital of Soochow University (No. JD- 


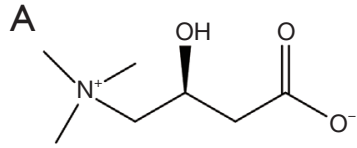

L-carnitine (target analyte)
B

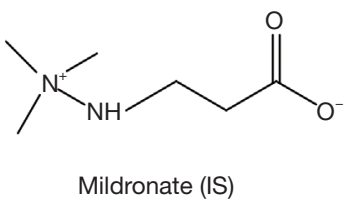

Figure 1 Chemical structures of analyte L-carnitine and mildronate. IS, internal standard.

LK-2020-038-01). All procedures performed in this study involving human participants were in accordance with the Declaration of Helsinki (as revised in 2013), and informed consent was taken from all the patients and healthy controls. Dialysis patients were recruited from the department of nephrology, while healthy controls were recruited from the medical examination department.

The inclusion criteria were as follows: aged 18-70 years, balanced age and gender distribution, no underlying diseases except kidney disease, and never having taken drugs that affect L-carnitine metabolism. The healthy control group was recruited from the physical examination department, and the inclusion criteria were based on the inclusion of the nephropathy group. The proportion of cases included in each age group was similar, as was the basic physical condition. The exclusion criteria were as follows: liver disease, cardiovascular disease, anemia, history of benign and malignant neoplasms, history of drug or alcohol abuse, vegetarian diet, or past use of meldonium.

There were 105 human participants recruited who had not taken L-carnitine, including 33 patients on hemodialysis, 33 patients on peritoneal dialysis, and 39 healthy people. Additionally, 39 patients on hemodialysis who had taken L-carnitine, were recruited. Blood samples were obtained before and after dialysis in the hemodialysis patients, while only blood samples after peritoneal dialysis were obtained from patients on peritoneal dialysis. At the time of sample collection, the proportion of stable hemodialysis patients meeting inclusion criteria was higher in males than in females. The clinical characteristics of the participants are shown in Table 1.

\section{Materials}

L-carnitine (purity >99.5\%) and MILD (purity >99.6\%) were purchased from Dalian Meilun Biotechnology Co., Ltd. (Dalian, China). Acetonitrile [(ACN); purity $>99.9 \%$ ] and formic acid [(FA); purity $>99.0 \%$ ] of liquid chromatography grade were obtained from Merck KGaA
(Darmstadt, Germany) and Anaqua Chemical Supply (Wilmington, DE, USA), respectively. Ammonium formate (purity $>99.0 \%$ ) was obtained from Shanghai Macklin Biochemical Co., Ltd. (Shanghai, China). Water was purified and deionized with a Milli-Q system manufactured by Millipore (Bedford, MA, USA).

\section{Instruments and conditions}

Chromatographic separation of the samples was carried out using a Dionex UltiMate 3000 Series UHPLC (Thermo Fisher Scientific, Waltham, MA, USA) equipped with an analytical column Hypersil GOLD HILIC column $(1.9 \mu \mathrm{m}, 100 \mathrm{~mm} \times 2.1 \mathrm{~mm}$; Thermo Fisher Scientific, USA). The mobile phase consisted of $0.1 \%(\mathrm{v} / \mathrm{v})$ formic acid and $10 \mathrm{mM}$ ammonium formate in water (solvent $\mathrm{A}$ ) and $\mathrm{ACN}$ (solvent $\mathrm{B}$ ). An isocratic elution with a flow rate of $0.2 \mathrm{~mL} / \mathrm{min}$ was performed in $50 \%$ of solvent $\mathrm{A}$ at room temperature $\left(25^{\circ} \mathrm{C}\right)$ and the total running time was $5 \mathrm{~min}$. Aliquots of $5 \mu \mathrm{L}$ of the sample extract were injected into the chromatographic system.

For the targeted analysis of plasma L-carnitine, the UHPLC system coupled to an Orbitrap mass spectrometer (Q Exactive ${ }^{\mathrm{TM}}$, Thermo Fisher Scientific, USA) were used. Operating with a heated electrospray interface (HESIII, Thermo Fisher Scientific, USA), in positive (ESI+) ionization mode was performed according to the following operational parameters: spray voltage, $3.8 \mathrm{kV}$; sheath gas $(\mathrm{N} 2,>95 \%)$ flow rate, 40; auxiliary gas $(\mathrm{N} 2,>95 \%)$ flow rate, 10; resolution, 70,000; S-lens RF level, 50; auxiliary gas heater temperature, $350^{\circ} \mathrm{C}$; and capillary temperature, $320^{\circ} \mathrm{C}$. The automatic gain control (AGC) was set at a target value of $1 \times 10^{6}$. The mass spectra were acquired in full scan mode and targeted-single ion monitoring (t-SIM) mode. Mass range in the full scan mode was set at $\mathrm{m} / \mathrm{z} 50-500$. In the t-SIM mode, the targeted mass of L-carnitine and mildronate were set at $\mathrm{m} / \mathrm{z} 162.11247$ and 147.11280, respectively. All the analyses were performed without lock mass. The data were collected and processed using Xcalibur ${ }^{\mathrm{TM}}$ version 2.2.1 (Thermo Fisher Scientific, Les Ulis, France) with Processing Setup, Qualitative and Quantitative browser. Genesis peak detection was applied.

\section{Preparation of stock and calibration standard solutions}

Totals of $10.58 \mathrm{mg}$ of L-carnitine and $10.10 \mathrm{mg}$ of MILD were accurately weighed using a one hundred thousandth scale, and then dissolved in $10 \mathrm{~mL}$ volumetric flask with 
Table 1 Clinical characteristics of patients on dialysis and healthy control

\begin{tabular}{|c|c|c|c|c|}
\hline Parameters & Healthy control (N=39) & Peritoneal dialysis $(\mathrm{N}=33)$ & \multicolumn{2}{|c|}{ Hemodialysis $(\mathrm{N}=72)$} \\
\hline Age $(Y)$ & $54.3 \pm 13.9$ & $54.6 \pm 17.5$ & \multicolumn{2}{|c|}{$58.4 \pm 15.5$} \\
\hline Gender (M/F) & $20 / 19$ & $17 / 16$ & \multicolumn{2}{|c|}{$45 / 27$} \\
\hline $\mathrm{Hb}(\mathrm{g} / \mathrm{L})$ & $143.2 \pm 10.4$ & $101.7 \pm 13.1$ & $116.6 \pm 15.9$ & - \\
\hline TRIG (mmol/L) & $0.9 \pm 0.4$ & $1.6 \pm 1.5$ & $1.7 \pm 1.1$ & - \\
\hline TC (mmol/L) & $4.7 \pm 0.6$ & $4.2 \pm 1.2$ & $3.7 \pm 1.0$ & - \\
\hline HDL-C (mmol/L) & $1.6 \pm 0.4$ & $0.9 \pm 0.4$ & $1.0 \pm 0.3$ & - \\
\hline LDL-C (mmol/L) & $2.7 \pm 0.4$ & $2.3 \pm 1.1$ & $2.0 \pm 0.7$ & - \\
\hline $\mathrm{HbA1c}(\%)$ & $5.2 \pm 0.3$ & $6.3 \pm 1.4$ & $6.2 \pm 1.2$ & - \\
\hline Urea (mmol/L) & $4.8 \pm 1.1$ & $19.6 \pm 8.1$ & $25.0 \pm 7.4$ & $8.4 \pm 3.7$ \\
\hline
\end{tabular}

Values are mean \pm standard deviation. "-" represents that no corresponding examination after hemodialysis. Hb, hemoglobin; Cre, creatinine; TRIG, triglycerides; TC, total cholesterol; HDL-C, high density lipoprotein cholesterol; LDL-C, low density lipoprotein cholesterol; UA, uric acid; FPG, fasting plasma glucose; HbA1c, glycosylated hemoglobin.

deionized water, respectively. Afterwards, the stock solutions of L-carnitine and MILD $(1 \mathrm{mg} / \mathrm{mL})$ were prepared in deionized water. The calibration standards of L-carnitine were prepared by appropriate dilution of the stock solution with deionized water, and the concentrations of standards were set at $6.169,12.337,24.674,49.348,74.023,98.697$, and $197.394 \mu \mathrm{M}$, while a final concentration of $10 \mu \mathrm{g} / \mathrm{mL}$ MILD was prepared by diluting the MILD stock solution with deionized water and used as the IS working solution. Correspondingly, quality control (QC) samples for lower limit of quantification (LLOQ), low QC (QL), mid QC $(\mathrm{QM})$, and high $\mathrm{QC}(\mathrm{QH})$ were $6.169,18.506,49.349$, and $154.216 \mu \mathrm{M}$. All solutions were frozen at $-80{ }^{\circ} \mathrm{C}$ prior to use. Calibration and QC samples were prepared by $50 \mu \mathrm{L}$ of raw human plasma spiked with an appropriate amount of the analytes $(5 \mu \mathrm{L})$ and $\mathrm{IS}(5 \mu \mathrm{L})$ solutions.

\section{Sample preparation}

For the extraction of plasma L-carnitine, $5 \mu \mathrm{L}$ IS working solution and $5 \mu \mathrm{L}$ deionized water were added to $50 \mu \mathrm{L}$ plasma sample and mixed thoroughly. After adding $450 \mu \mathrm{L}$ $\mathrm{ACN}$, the mixture was vortexed thoroughly and then centrifuged at $13,000 \times \mathrm{g}$ for $10 \mathrm{~min}$ at $4{ }^{\circ} \mathrm{C}$. The obtained supernatant was further centrifuged and the second supernatant was collected and diluted 10 times with ACN, then analyzed using UHPLC-Orbitrap-HRMS. Blood samples from each participant were processed in the same way 3 times.

\section{Method validation}

\section{Selectivity and residual effect}

We prepared 6 batches of double blank plasma (no $\mathrm{L}$-carnitine or IS was added to the plasma, $10 \mu \mathrm{L} \mathrm{H}_{2} \mathrm{O}$ was added instead), single blank plasma (no L-carnitine was added to the plasma, $5 \mu \mathrm{L} \mathrm{H} \mathrm{H}_{2} \mathrm{O}$ and $5 \mu \mathrm{L}$ IS was added instead), LLOQ, and upper limit of quantification (ULOQ) were prepared to investigate the selectivity and residual effect. Those plasma samples were processed following the sample procedure described above. Blank samples were injected before LLOQ and right after ULOQ.

\section{Accuracy and precision}

To assess the accuracy and precision of this method, QC samples were tested at 4 different concentrations in 3 separate validation runs. The precision was expressed as the relative standard deviation (RSD\%), within-run and between-run precisions were calculated with the following equation: 


$$
\mathrm{RSD} \%=\frac{\mathrm{SD}}{\text { Mean }} \times 100
$$

where Mean is the mean of the actual measured concentrations and SD is the standard deviation of the measured concentrations.

Accuracy was defined as the percent relative error (RE\%) and was calculated as follows:

$$
\mathrm{RE} \%=\frac{\mathrm{M}-\mathrm{T}}{\mathrm{T}} \times 100
$$

where $M$ is the measured concentration and $\mathrm{T}$ is the theoretical concentration.

\section{Matrix effect and extraction recovery}

For the determination of matrix effect, firstly, the normalized area ratios of $3 \mathrm{~L}$-carnitine levels (QH, QM, QL) spiked in 6 different human plasma and that of the same amounts dissolved in the same volume of $\mathrm{H}_{2} \mathrm{O}$ were compared, and both of them followed the same procedure described above. The normalized area ratio was calculated using the peak area of the molecular target divided by that of IS. Secondly, the calibration curves of spiked plasma and spiked water samples were created (15). The extent of the matrix effect was assessed by comparing slopes of calibration curves prepared in spiked plasma and spiked water samples, which was calculated as follows:

$$
\mathrm{MF} \%=100-\left(\mathrm{k}_{\text {spiked }} / \mathrm{k}_{\text {solvent }} \times 100\right)
$$

where MF is the matrix effect, $k$ is the slope of calibration curve.

The extraction recovery from plasma was calculated by the difference between normalized area ratios of pre-spiked plasma (adding standards to the plasma before extraction) and post-spiked plasma (adding standards to the ACN extract of plasma). Similarly, the experiment was conducted with 6 batches of human plasma, each pre- or post-spiked matrix was spiked with 3 levels of L-carnitine.

\section{Fortified validation}

The experiment was conducted on six separate occasions using different matrices, the calibration curve and the corresponding single blank plasma samples and QC samples were prepared each time. The normalized area ratio of each single blank plasma sample was then substituted into the calibrations conducted with 6 matrices to acquire the calculated concentrations and then compare the difference between these results. The difference was measured by precision.

\section{Stability testing}

To investigate the stability, 5 batches of QL and $\mathrm{QH}$ samples were stored at room temperature, $4{ }^{\circ} \mathrm{C}$, and -80 ${ }^{\circ} \mathrm{C}$ for 1 week and thawed in 3 freeze-thaw cycles. The corresponding measured concentrations were compared with that of samples which analyzed directly after extraction.

\section{Statistical analysis}

One-way analysis of variance (ANOVA) was used to compare the differences of plasma L-carnitine levels among groups, and the results were expressed as mean \pm standard deviation $(\bar{x} \pm \mathrm{SD})$. Statistical analyses were conducted by GraphPad Prism 6.0 statistical software (GraphPad Software, La Jolla, CA, USA), all the analyses were 2-sided, and $\mathrm{P}<0.05$ was considered statistically significant.

\section{Results}

\section{Selectivity and residual effect}

The typical chromatograms and spectra of L-carnitine and MILD in plasma samples are shown in Figure 2. The typical mass spectrum of L-carnitine is shown in Figure $2 \mathrm{~A}$ and that of IS is shown in Figure $2 B$. The chromatograms of L-carnitine and IS in the LLOQ and 2 blank plasma samples, are shown in Figure $2 C$ and Figure 2D, respectively. There were no interfering peaks observed near the retention time of interest, which meant that the developed method had sufficient selectivity and specificity to detect plasma L-carnitine. The result also showed the response of LLOQ was slightly higher than that of endogenous L-carnitine in the blank plasma, and there was no residue of L-carnitine and IS after the injection of ULOQ.

\section{Linearity, accuracy, and precision}

Normal content of plasma endogenous L-carnitine is approximately 40-50 $\mu \mathrm{M}$ (28). Thus, the final concentration range of standard solutions in plasma was set at the level of 6.200-198.386 $\mu$ M. Besides, the result of the corresponding blank plasma was taken into consideration when constructing the calibration function. The calibration curves were constructed by a weighted linear regression model with a weighting factor of $1 / \chi^{2}$. The relative peak area ratio of the target analyte and the IS was plotted against the L-carnitine concentration. The lowest concentration for the calibration curve was considered to 

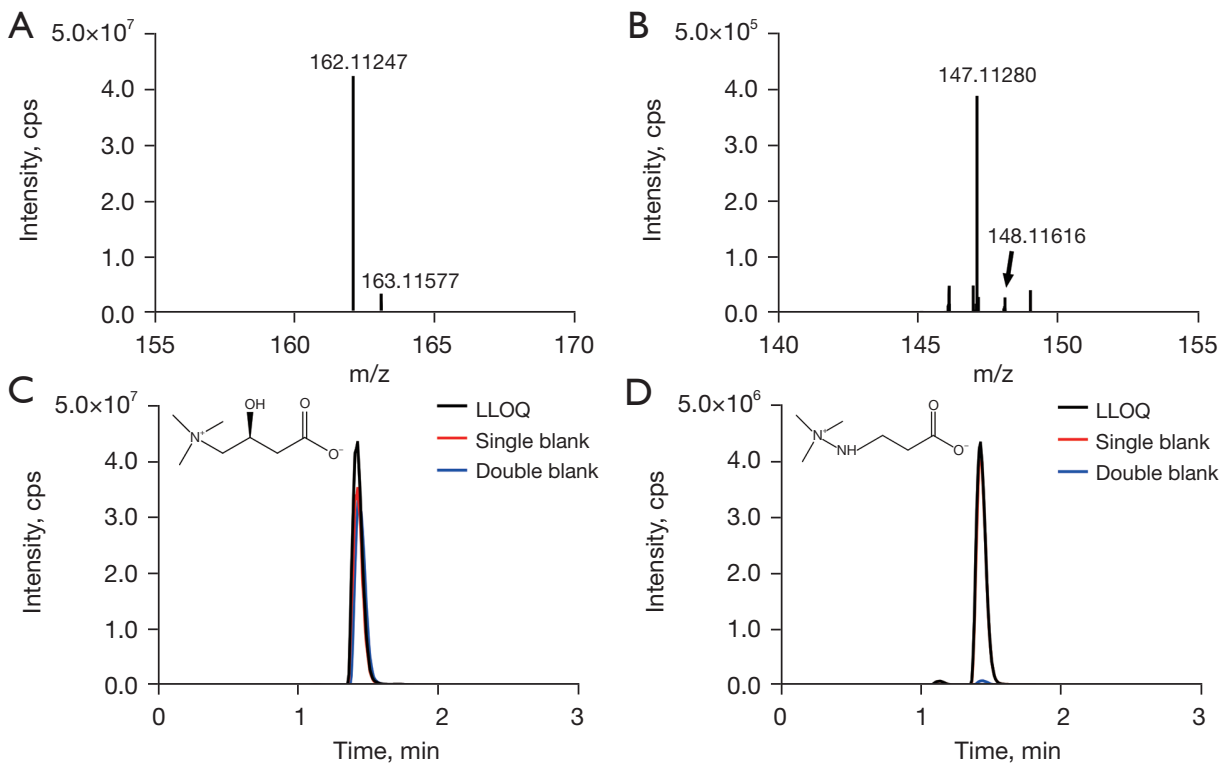

Figure 2 The chromatograms and spectra of LC and MILD in plasma sample. Exact characteristic mass-to-charge ratio of LC (A) and MILD (B) and target-SIM spectra of LC (C) and MILD (D) in plasma. Single blank: no LC was added to the plasma, $5 \mu \mathrm{L} \mathrm{H}_{2} \mathrm{O}$ and $5 \mu \mathrm{L}$ IS was added instead; double blank: no LC or IS was added to the plasma, $10 \mu \mathrm{L} \mathrm{H}_{2} \mathrm{O}$ was added instead. LC, L-carnitine; MILD, mildronate; cps, counts per second; IS, internal standard; LLOQ, lower limit of quantification.

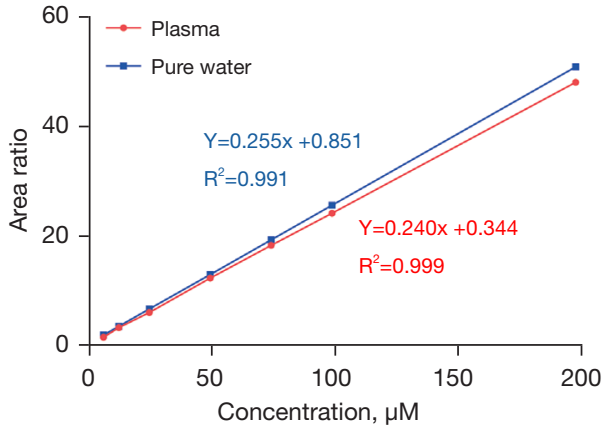

Figure 3 Calibration curves prepared in spiked plasma and spiked water samples. The plasma endogenous L-carnitine was subtracted when constructed the curve.

be the low limit of quantitation (LLOQ) (Figure 2), and good linearity was observed $\left(\mathrm{R}^{2}=0.9913\right.$, Figure $\left.\mathrm{S} 1\right)$. The within-run and between-run precisions and the accuracy obtained in the study were better than prescribed by the acceptance criteria (FDA/CDER, 2003), both accuracy and precision were $\leq \pm 15 \%$ (LLOQ, $\leq \pm 20 \%$ ) (Table S1).

\section{Matrix effect and recovery}

The matrix factor (MF) was calculated by the ratio of the L-carnitine peak area in the presence of matrix and that in deionized water. The IS normalized MF of QC samples in 6 matrices ranged from $91.803 \%$ to $99.862 \%$ compared to non-matrix ones. The result illustrated that the ion suppression in different plasma is stable and mild, with RSD ranging from $0.715 \%$ to $9.867 \%$. From the slopes comparison, it was observed that the plasma matrices diminished the response of the analytes by $5.882 \%$ (Figure 3).

Unexpectedly high extraction recoveries were found following the above procedure, that of QL, QM, and $\mathrm{QH}$ were $154.416 \%, 123.053 \%$, and $102.945 \%$, with RSDs of $3.593 \%, 5.745 \%$, and $6.057 \%$, respectively (Table S2). Besides, this paper lists four sample processing methods, and compares the recovery values of different processing methods (Figure S2 and Tables S3,S4). It can be seen that the high recoveries of this method were caused by the different adding time of IS.

\section{Fortified validation}

To further validate the developed method, we carried out a fortified validation assay, which contained 6 calibration curves constructed with different matrix (plasma A - plasma F). The precision of plasma $\mathrm{A}-\mathrm{F}$ detection in this assay 


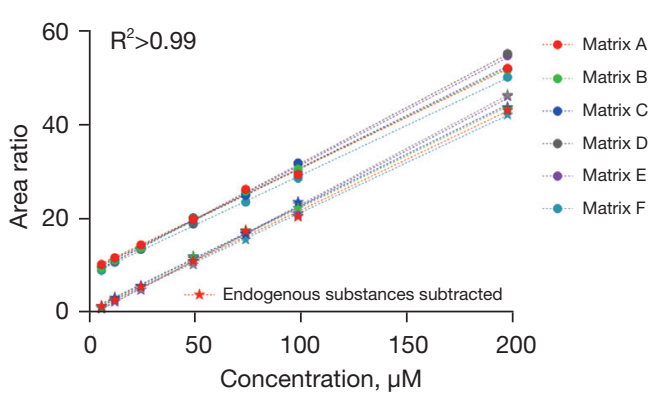

Figure 4 Six calibration curves of L-carnitine using six different plasma matrix. Dots represent initial standard curves containing endogenous level of L-carnitine, while stars represent the endogenous substances subtracted calibration curves of L-carnitine in the plasma.

was $5.145-8.737 \%$, which reflected good precision and confirmed the reliability of the assay developed here. It was further shown that the interference with results was caused by the matrix effect is minimal by this method. The results are listed in detail in Figure 4 and Table S5.

\section{Stability}

L-carnitine in plasma remained stable for 1 week at room temperature. Storage of plasma samples at 4 and $-80^{\circ} \mathrm{C}$ also showed a high stability for 1 week. For further validation of the stability of L-carnitine, thawing in 3 freeze-thaw cycles had a slight impact on the response of analytes (Figure S3).

\section{Quantification of plasma endogenous L-carnitine in patients on dialysis}

Using the method developed above, the content of L-carnitine in plasma samples was detected (Figures 5,6, and Figure S4). The average value of each participant's 3 tests was taken as the final measured value. As Figure $5 \mathrm{~A}$ shows, the amount of L-carnitine in peritoneal dialysis patients $(34.35 \pm 3.065 \mu \mathrm{M}, \mathrm{n}=33)$ was lower than that in the normal control group $(40.88 \pm 1.238 \mu \mathrm{M}, \mathrm{n}=39)$, and it was statistically different $(\mathrm{P}=0.0402)$. It is worth noting that 20 out of 33 patients on peritoneal dialysis had different degrees of L-carnitine deficiency (Figure 6). Although $48.5 \%$ of these patients faced an extreme lack of L-carnitine (lower than $30 \mu \mathrm{M}$ ), the remainder still had acceptable levels of L-carnitine.

Furthermore, comparison between healthy participants and different groups of hemodialysis patients showed a significant difference (Figure 5B and Figure S4). The plasma L-carnitine level of pre-hemodialysis patients without L-carnitine supplementation $(29.16 \pm 2.243 \mu M, n=33)$ was notably lower than that of pre-hemodialysis patients with L-carnitine supplementation $(131.1 \pm 13.90 \mu M, n=39)$ and healthy participants. Although the average L-carnitine level of pre-hemodialysis patients who had taken the L-carnitine supplementation as a routine was notably higher than normal level, a quarter of those patients (9/39) still had different degrees of L-carnitine deficiency. In addition, the plasma L-carnitine concentrations of post-hemodialysis patients with or without L-carnitine supplementation were significantly lower than normal level, which were $31.41 \pm 3.409$ and $7.856 \pm 0.6442 \mu \mathrm{M}$, respectively. The average filtration rate of $\mathrm{L}$-carnitine in hemodialysis was approximately $74 \%$ here. The results in hemodialysis patients were consistent with previous studies $(3,29)$. Compared with the $100 \%$ extreme L-carnitine deficiency after dialysis without medication, the rate of L-carnitine deficiency after dialysis with medication was reduced to $81.8 \%(27 / 33)$ and $2 / 3$ of them experienced extreme deficiency $(21 / 33)$.

\section{Discussion}

L-carnitine is a low molecular weight hydrophilic substance which can be excreted by kidneys and easily lost via dialysis. In healthy people, L-carnitine deficiency is rare because it can be adequately supplied through diet and biosynthesis. However, deficiency is often observed in dialysis patients.

Several researches have declared that L-carnitine plays an important role in improving a variety of dialysis-related symptoms. Recent studies showed that low concentrations of L-carnitine would increase plasma B-type natriuretic peptide (BNP) levels in hemodialysis dialysis patients by affecting the activity of carnitine palmitoyltransferase 2 (CPT2) in cardiomyocytes (30). Additionally, low L-carnitine levels were associated with peripheral arterial stiffness in patients undergoing kidney transplantation (31). L-carnitine supplementation may improve erythropoietinresistant anemia, reduce muscle cramping, improve hemoglobin levels and cardiac function in patients on hemodialysis/peritoneal dialysis $(32,33)$.

Taken the clinical impacts of absolute L-carnitine deficiency and the huge difference in the deficiency degree of different dialysis situations into consideration, there is an eagerness to seek a means to promote precision treatment and rational drug use, reduce the waste of social resources 

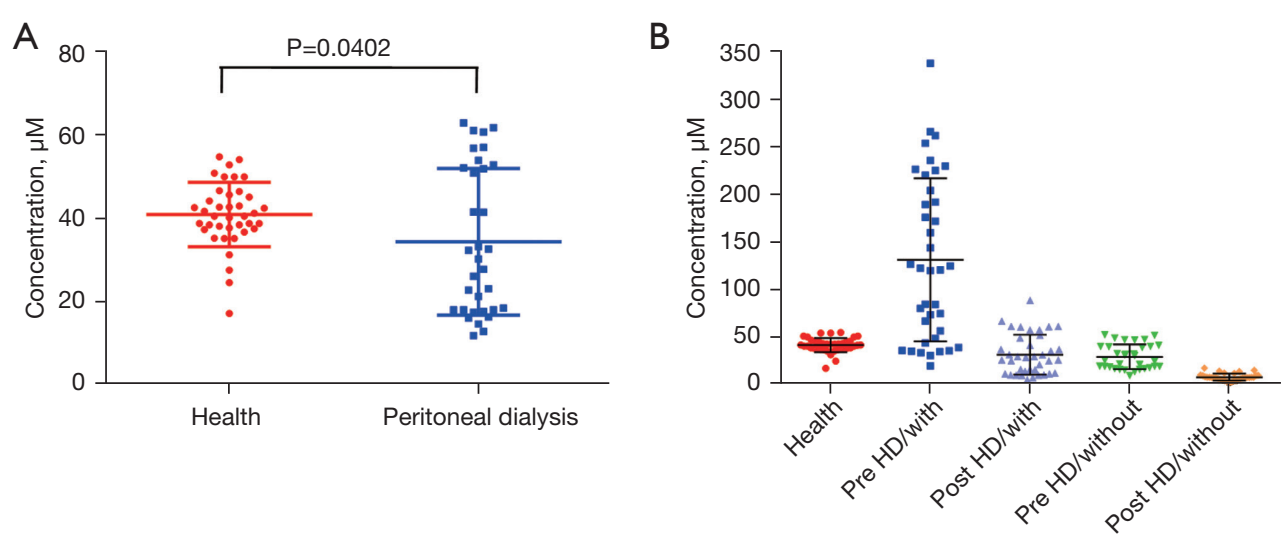

Figure 5 Plasma L-carnitine concentrations of healthy subjects and patients on dialysis. (A) Comparison between healthy subjects and patients with peritoneal dialysis, all of them had never taken L-carnitine, (B) comparison between healthy subjects and different groups of patients with hemodialysis. Patients with HD were divided into four groups according to pre/post HD and whether they were treated with $\mathrm{L}$-carnitine or not; $\mathrm{P}<0.05$ is considered statistically different. HD, hemodialysis.

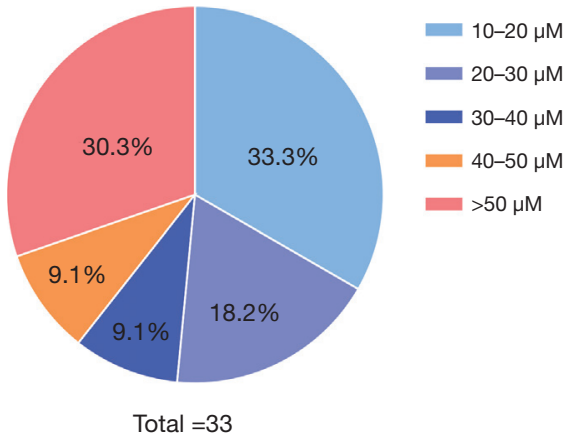

Figure 6 Distribution of plasma L-carnitine concentrations in peritoneal dialysis patients.

and the economic burden of patients.

Thus, this study developed and validated an UHPLCOrbitrap-MS method for rapid determination of plasma endogenous L-carnitine in dialysis patients. There was no need to prepare analyte-depleted biological matrix or artificial matrix, which simplified the analysis procedure and guaranteed the authenticity of the results. The results of the fortified validation showed that the L-carnitine level in each plasma sample could be accurately quantified using the ameliorated standard curve made from anyone's untreated plasma. Taken the cost of the annual warranty of the instrument, mobile phase and standard solutions into account, the total cost is then divided by the number of tests per year, which is the cost of testing per sample. In our hospital, the cost of using UHPLC-Orbitrap-HRMS to detect L-carnitine was about $¥ 50-100$ per sample.
To our knowledge, there are few published reports on the supplementation of L-carnitine in patients undergoing peritoneal dialysis $(5,32)$. Nevertheless, the results above declared that peritoneal dialysis patients without L-carnitine supplementation should pay more attention to the monitoring of L-carnitine concentration, due to the wide concentration range in the plasma of these patients. The majority of them were facing different degrees of L-carnitine deficiency, and half of them had an extreme lack of L-carnitine. Meanwhile, $39.4 \%$ of peritoneal dialysis patients had adequate plasma L-carnitine without additional supplementation. Drug abuse in these patients should be avoided in clinical treatment to reduce their medical burden. Additionally, all hemodialysis patients were advised to be routinely given a full dose of L-carnitine, no matter whether they had ever taken L-carnitine or not. All the hemodialysis patients without L-carnitine supplementation faced extreme L-carnitine deficiency, while the ones with L-carnitine supplementation still had a high risk of L-carnitine deficiency.

A number of limitations need to be further discussed in this study. First of all, the sample size of different groups was small, and these results require further verification through an expanded sample size. Secondly, the basic conditions of population included in the study have not been completely consistent between the groups, and it is unknown whether the inconsistency of these parameters will lead to the deviation of the results. Thirdly, the dialysis duration has not been stratified. The deficiency of L-carnitine may become more serious with the increase 
of dialysis years, which makes routine supplement of L-carnitine for peritoneal dialysis patients reasonable and necessary after reaching a certain number of dialysis years. Hence, a more detailed study on the rationality of L-carnitine supplementation in peritoneal dialysis patients according to various parameters is worthwhile.

\section{Conclusions}

A simple, rapid and precise UHPLC-Orbitrap-HRMS method for detection of endogenous L-carnitine was developed in this study, and the present results of plasma endogenous L-carnitine in dialysis patients indicated that the peritoneal dialysis patients were more deserving of a content test before the use of L-carnitine, which could be useful to promote rational drug use in future clinical practice.

\section{Acknowledgments}

Funding: This research was supported by the Soochow Science and Technology Project (SYSD2019182 and SYSD2020184), the Youth Project of Soochow Scientific Research Project (KJXW2019017), the Pre-Research Project of The Second Affiliated Hospital of Soochow University (SDFEYQN1917), Tianqing Pharmaceutical Foundation of Jiangsu Pharmaceutical Association (TQ2021058), Hengrui Pharmaceutical Foundation of Jiangsu Pharmaceutical Association (H202145), National Natural Science Foundation of China (82070838), and Lift Project for Discipline Construction of The Second Affiliated Hospital of Soochow University (XKTJXK202010).

\section{Footnote}

Reporting Checklist: The authors have completed the MDAR reporting checklist. Available at https://atm.amegroups. com/article/view/10.21037/atm-21-6784/rc

Data Sharing Statement: Available at https://atm.amegroups. com/article/view/10.21037/atm-21-6784/dss

Conflicts of Interest: All authors have completed the ICMJE uniform disclosure form (available at https:// atm.amegroups.com/article/view/10.21037/atm-216784/coif). All authors report funding from the Soochow Science and Technology Project (SYSD2019182 and
SYSD2020184), the Youth Project of Soochow Scientific Research Project (KJXW2019017), the pre-research project of the Second Affiliated Hospital of Soochow University (SDFEYQN1917), Tianqing Pharmaceutical Foundation of Jiangsu Pharmaceutical Association (TQ2021058), Hengrui Pharmaceutical Foundation of Jiangsu Pharmaceutical Association (H202145), National Natural Science Foundation of China (82070838), and Lift Project for Discipline Construction of The Second Affiliated Hospital of Soochow University (XKTJ-XK202010). The authors have no other conflicts of interest to declare.

Ethical Statement: The authors are accountable for all aspects of the work in ensuring that questions related to the accuracy or integrity of any part of the work are appropriately investigated and resolved. This study was approved by the Ethics Committee of The Second Affiliated Hospital of Soochow University (No. JDLK-2020-038-01). Informed consent was taken from all the patients and healthy controls. All procedures performed in this study involving human participants were in accordance with the Declaration of Helsinki (as revised in 2013).

Open Access Statement: This is an Open Access article distributed in accordance with the Creative Commons Attribution-NonCommercial-NoDerivs 4.0 International License (CC BY-NC-ND 4.0), which permits the noncommercial replication and distribution of the article with the strict proviso that no changes or edits are made and the original work is properly cited (including links to both the formal publication through the relevant DOI and the license). See: https://creativecommons.org/licenses/by-nc-nd/4.0/.

\section{References}

1. Longo A, Bruno G, Curti S, et al. Determination of L-carnitine, acetyl-L-carnitine and propionyl-Lcarnitine in human plasma by high-performance liquid chromatography after pre-column derivatization with 1-aminoanthracene. J Chromatogr B Biomed Appl 1996;686:129-39.

2. Mingrone G, Greco AV, Capristo E, et al. L-carnitine improves glucose disposal in type 2 diabetic patients. J Am Coll Nutr 1999; 18:77-82.

3. Evans AM, Faull RJ, Nation RL, et al. Impact of hemodialysis on endogenous plasma and muscle carnitine levels in patients with end-stage renal disease. Kidney Int 2004;66:1527-34. 
4. Calò LA, Vertolli U, Davis PA, et al. L carnitine in hemodialysis patients. Hemodial Int 2012;16:428-34.

5. Eknoyan G, Latos DL, Lindberg J, et al. Practice recommendations for the use of L-carnitine in dialysisrelated carnitine disorder. National Kidney Foundation Carnitine Consensus Conference. Am J Kidney Dis 2003;41:868-76.

6. Bellinghieri G, Santoro D, Calvani M, et al. Carnitine and hemodialysis. Am J Kidney Dis 2003;41:S116-22.

7. Fathizadeh H, Milajerdi A, Reiner $\check{Z}$, et al. The effects of L-carnitine supplementation on indicators of inflammation and oxidative stress: a systematic review and meta-analysis of randomized controlled trials. J Diabetes Metab Disord 2020;19:1879-94.

8. Hamedi-Kalajahi F, Imani H, Mojtahedi S, et al. Effect of L-Carnitine Supplementation on Inflammatory Markers and Serum Glucose in Hemodialysis Children: A Randomized, Placebo-Controlled Clinical Trial. J Ren Nutr 2021. [Epub ahead of print].

9. Fatouros IG, Douroudos I, Panagoutsos S, et al. Effects of L-carnitine on oxidative stress responses in patients with renal disease. Med Sci Sports Exerc 2010;42:1809-18.

10. Morand R, Donzelli M, Haschke M, et al. Quantification of plasma carnitine and acylcarnitines by high-performance liquid chromatography-tandem mass spectrometry using online solid-phase extraction. Anal Bioanal Chem 2013;405:8829-36.

11. Minkler PE, Stoll MS, Ingalls ST, et al. Quantification of carnitine and acylcarnitines in biological matrices by HPLC electrospray ionization-mass spectrometry. Clin Chem 2008;54:1451-62.

12. van der Heeft E, Bolck YJ, Beumer B, et al. Full-scan accurate mass selectivity of ultra-performance liquid chromatography combined with time-of-flight and orbitrap mass spectrometry in hormone and veterinary drug residue analysis. J Am Soc Mass Spectrom 2009;20:451-63.

13. Gómez-Pérez ML, Plaza-Bolaños P, Romero-González $\mathrm{R}$, et al. Comprehensive qualitative and quantitative determination of pesticides and veterinary drugs in honey using liquid chromatography-Orbitrap high resolution mass spectrometry. J Chromatogr A 2012;1248:130-8.

14. Concheiro M, Castaneto M, Kronstrand R, et al. Simultaneous determination of 40 novel psychoactive stimulants in urine by liquid chromatography-high resolution mass spectrometry and library matching. J Chromatogr A 2015;1397:32-42.

15. Isaguirre AC, Olsina RA, Martinez LD, et al. Rapid and sensitive HILIC-MS/MS analysis of carnitine and acetylcarnitine in biological fluids. Anal Bioanal Chem 2013;405:7397-404.

16. Panuwet P, Hunter RE Jr, D'Souza PE, et al. Biological Matrix Effects in Quantitative Tandem Mass Spectrometry-Based Analytical Methods: Advancing Biomonitoring. Crit Rev Anal Chem 2016;46:93-105.

17. Gachet MS, Rhyn P, Bosch OG, et al. A quantitiative LCMS/MS method for the measurement of arachidonic acid, prostanoids, endocannabinoids, $\mathrm{N}$-acylethanolamines and steroids in human plasma. J Chromatogr B Analyt Technol Biomed Life Sci 2015;976-977:6-18.

18. Jing Z, Kuang L, Liu N, et al. LC-MS/MS for the simultaneous determination of polar endogenous ADMA and $\mathrm{CML}$ in plasma and urine from diabetics. Bioanalysis 2015;7:1261-71.

19. Scherer M, Gnewuch C, Schmitz G, et al. Rapid quantification of bile acids and their conjugates in serum by liquid chromatography-tandem mass spectrometry. J Chromatogr B Analyt Technol Biomed Life Sci 2009;877:3920-5.

20. Oka K, Yamamoto M, Nonaka T, et al. The significance of artificial cerebrospinal fluid as perfusate and endoneurosurgery. Neurosurgery 1996;38:733-6.

21. Jiang W, Liu L, Chen Y. Simultaneous Detection of Human C-Terminal p53 Isoforms by Single Template Molecularly Imprinted Polymers (MIPs) Coupled with Liquid Chromatography-Tandem Mass Spectrometry (LC-MS/MS)-Based Targeted Proteomics. Anal Chem 2018;90:3058-66.

22. Goodenough AK, Onorato JM, Ouyang Z, et al. Quantification of 4-beta-hydroxycholesterol in human plasma using automated sample preparation and LC-ESIMS/MS analysis. Chem Res Toxicol 2011;24:1575-85.

23. Ongay S, Hendriks G, Hermans J, et al. Quantification of free and total desmosine and isodesmosine in human urine by liquid chromatography tandem mass spectrometry: a comparison of the surrogate-analyte and the surrogatematrix approach for quantitation. J Chromatogr A 2014;1326:13-9.

24. Thakare R, Chhonker YS, Gautam N, et al. Quantitative analysis of endogenous compounds. J Pharm Biomed Anal 2016;128:426-37.

25. Pidpruzhnykov YV, Sabko VE, Iurchenko VV, et al. UPLCMS/MS method for bioequivalence study of oral drugs of meldonium. Biomed Chromatogr 2012;26:599-605.

26. Yan S, Wang X, Yang C, et al. Insights Into Walnut Lipid Metabolism From Metabolome and Transcriptome Analysis. Front Genet 2021;12:715731. 
27. Geng J, Xiao L, Chen C, et al. An integrated analytical approach based on enhanced fragment ions interrogation and modified Kendrick mass defect filter data mining for in-depth chemical profiling of glucosinolates by ultrahigh-pressure liquid chromatography coupled with Orbitrap high resolution mass spectrometry. J Chromatogr A 2021;1639:461903.

28. Evans A. Dialysis-related carnitine disorder and levocarnitine pharmacology. Am J Kidney Dis 2003;41:S13-26.

29. Evans AM, Faull R, Fornasini G, et al. Pharmacokinetics of L-carnitine in patients with end-stage renal disease undergoing long-term hemodialysis. Clin Pharmacol Ther 2000;68:238-49.

30. Sugiyama M, Hazama T, Nakano K, et al. Effects of Reducing L-Carnitine Supplementation on Carnitine

Cite this article as: Jiang WT, Tang LX, Pan J, Su CJ, Shen Z, $\mathrm{Hu} \mathrm{ZH}$, Zhan ZB, Shi AM. Development and validation of an UHPLC-Orbitrap-HRMS method for rapid determination of endogenous L-carnitine in patients on hemodialysis/peritoneal dialysis and its application to promote rational drug use. Ann Transl Med 2022;10(2):103. doi: 10.21037/atm-21-6784
Kinetics and Cardiac Function in Hemodialysis Patients: A Multicenter, Single-Blind, Placebo-Controlled, Randomized Clinical Trial. Nutrients 2021;13:1900.

31. Lai YH, Lee MC, Ho GJ, et al. Association of Low Serum 1-Carnitine Levels with Peripheral Arterial Stiffness in Patients Who Undergo Kidney Transplantation. Nutrients 2019;11:2000.

32. Kaneko S, Yanai K, Kitano T, et al. Change in Anemia by Carnitine Supplementation in Patients Undergoing Peritoneal Dialysis: A Retrospective Observational Study. Front Med (Lausanne) 2021;8:767945.

33. Kuwasawa-Iwasaki M, Io H, Muto M, et al. Effects of L-Carnitine Supplementation in Patients Receiving Hemodialysis or Peritoneal Dialysis. Nutrients 2020;12:3371. 


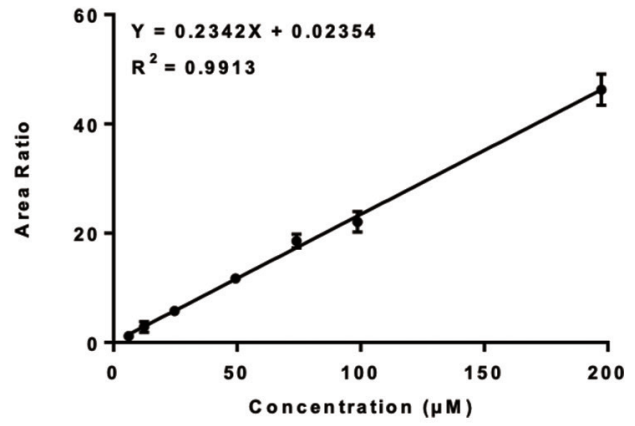

Figure S1 Endogenous substances subtracted calibration curve of L-carnitine in the plasma.

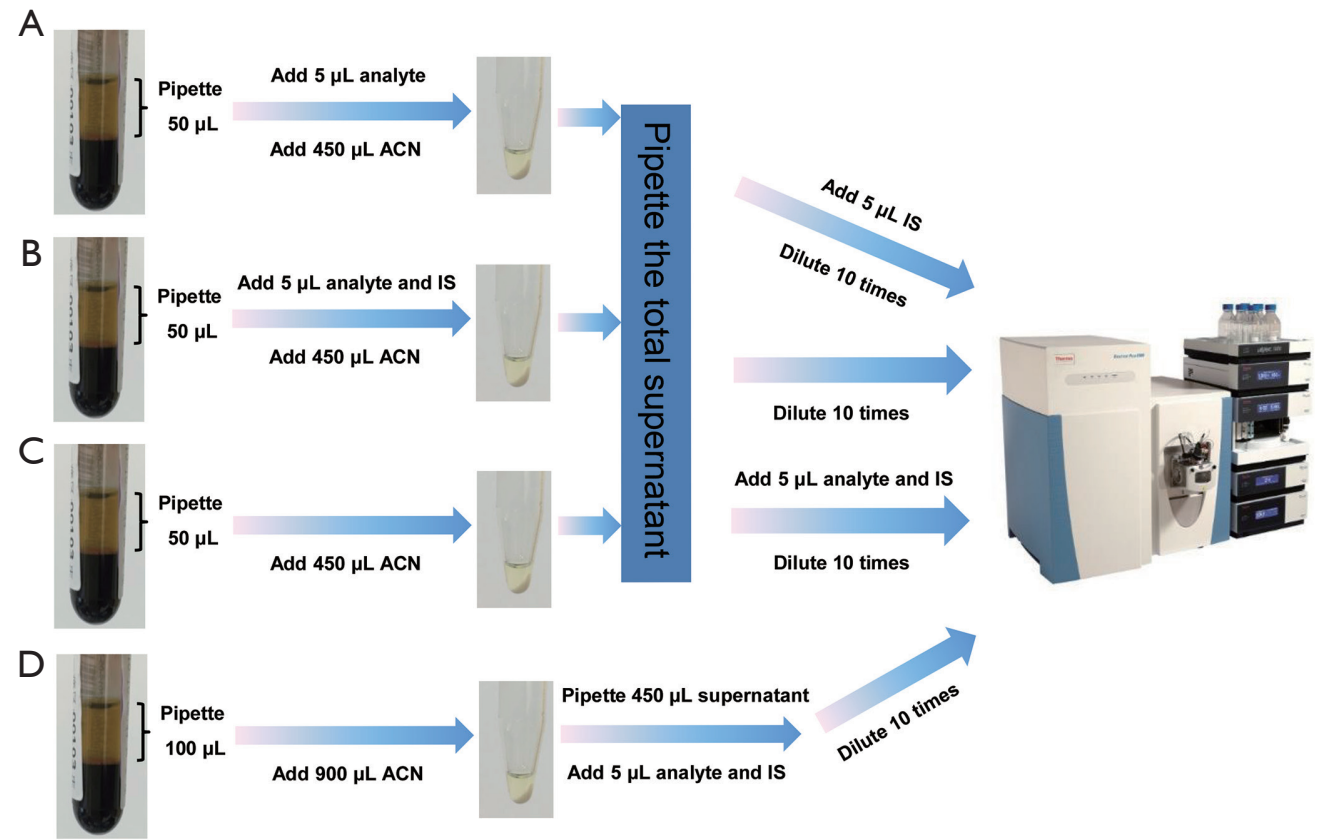

Figure S2 Different sample processing methods. IS, internal standard; ACN, Acetonitrile. 


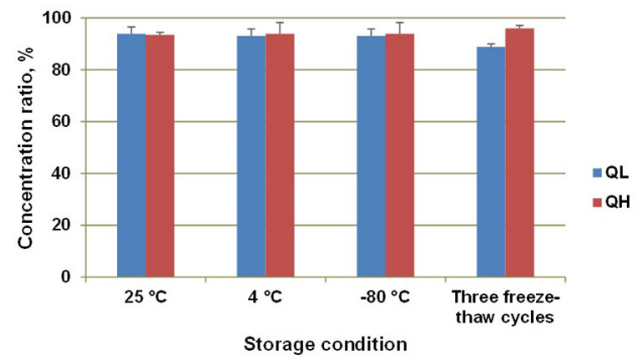

Figure S3 Assessment of stability of the analyte L-carnitine. QL, low concentration quality control sample; QH, high concentration quality control sample.
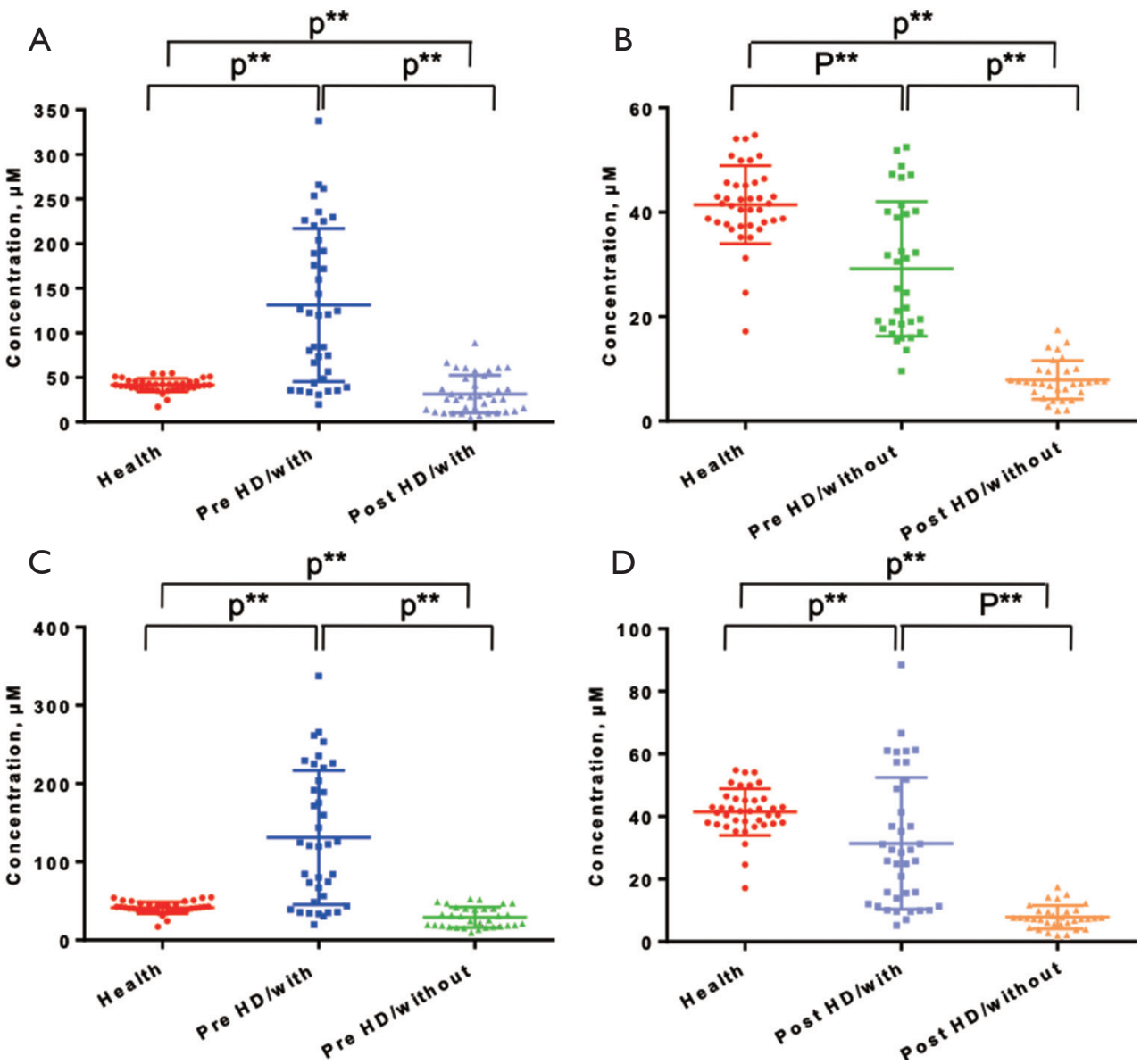

Figure S4 Statistical differences between healthy subjects and different HD groups. Patients with HD were divided into 4 groups according to pre/post HD and whether they were treated with $\mathrm{L}$-carnitine or not. ${ }^{*}, \mathrm{P}<0.01$ is considered to indicate a statistically significant difference. HD, hemodialysis. 
Table S1 Intra-day and inter-day precision and accuracy for QC samples

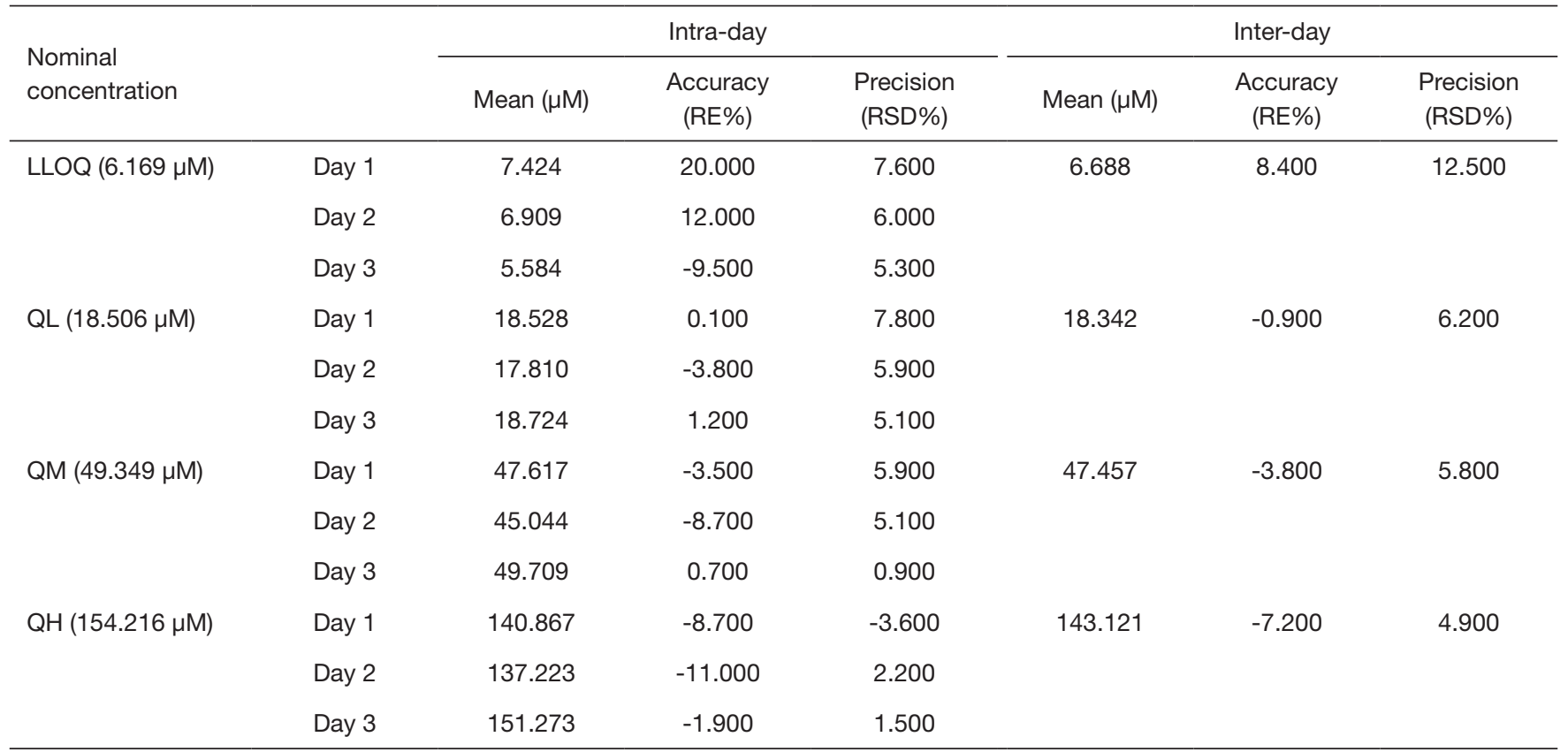

QC samples, quality control samples; LLOQ, lower limit of quantification; QL, low concentration QC; QM, middle concentration QC; QH, high concentration $\mathrm{QC}$; RE, relative error; $\mathrm{RSD}$, relative standard deviation.

Table S2 The matrix factor and recovery of the method developed

\begin{tabular}{lcccc}
\hline \multirow{2}{*}{ QC samples } & \multicolumn{2}{c}{ Matrix factor (\%) } & \multicolumn{2}{c}{ Recovery (\%) } \\
\cline { 2 - 5 } & Mean & RSD & Mean & RSD \\
\hline QL & 91.803 & 9.867 & 154.416 & 3.593 \\
QM & 96.229 & 3.510 & 123.053 & 5.745 \\
QH & 99.862 & 0.715 & 102.945 & 6.057 \\
\hline
\end{tabular}

QC samples, quality control samples; LLOQ, lower limit of quantification; QL, low concentration QC; QM, middle concentration QC; QH, high concentration QC; RSD, relative standard deviation.

Table S3 The area ratio of different sample processing methods

\begin{tabular}{lccc}
\hline \multirow{2}{*}{ Sample ID } & \multicolumn{2}{c}{ Response intensity } & Area ratio (AR) \\
\cline { 2 - 3 } & L-carnitine & MILD & \multirow{2}{*}{8.574} \\
QM-1 $^{\text {a }}$ & 31437052.889 & 3666389.647 & 20.876 \\
QM-2 $^{\text {b }}$ & 34716499.506 & 1662983.625 & 17.392 \\
QM-3 $^{\text {c }}$ & 58391600.575 & 3357444.474 & 17.214 \\
QM-4 $^{\text {d }}$ & 55451867.434 & 3221367.498 & 17
\end{tabular}

${ }^{\mathrm{a}} \mathrm{QM}-1$ is defined as the middle concentration quality control (QM) sample processed according to method A; ${ }^{\mathrm{b}} \mathrm{QM}-2$ is defined as the $\mathrm{QM}$ sample processed according to method B; ${ }^{C} \mathrm{QM}-3$ is defined as the QM sample processed according to method C; ${ }^{\text {d }} \mathrm{QM}-4$ is defined as the QM sample processed according to method D; MILD, mildronate. 
Table S4 The recovery comparison of different sample processing methods

\begin{tabular}{lcc}
\hline Recovery of QMs $(H / V)$ & $\mathrm{AR}_{\mathrm{QM}-3}$ & $\mathrm{AR}_{\mathrm{QM}-4}$ \\
\hline $\mathrm{AR}_{\mathrm{QM}-1}$ & $49.302 \%$ & $49.811 \%$ \\
$\mathrm{AR}_{\mathrm{QM}-2}$ & $120.035 \%$ & $121.275 \%$ \\
\hline
\end{tabular}

${ }^{*} \mathrm{H} / \mathrm{V}$ is defined as the ratio of the horizontal values and the vertical values and the italic fonts represents the recovery of the method developed here; AR, area ratio; $\mathrm{QM}-1$ is defined as the $\mathrm{QM}$ middle concentration quality control (QM) sample processed according to method A; QM-2 is defined as the QM sample processed according to method B; QM-3 is defined as the QM sample processed according to method C; QM-4 is defined as the QM sample processed according to method D.

Table S5 The fortified validation of calculated concentrations of the endogenous L-carnitine with 6 different calibration curves

\begin{tabular}{lcccccccc}
\hline \multirow{2}{*}{$\begin{array}{l}\text { Calculated } \\
\text { concentration }(\mu \mathrm{M})\end{array}$} & \multicolumn{9}{c}{ Calibration curves with corresponding matrix } & Mean & RSD (\%) \\
\cline { 2 - 7 } & $\mathrm{A}$ & $\mathrm{B}$ & $\mathrm{C}$ & $\mathrm{D}$ & $\mathrm{E}$ & $\mathrm{F}$ & \\
\hline Blank-plasma A & 40.587 & 39.055 & 38.165 & 44.166 & 44.955 & 45.960 & 42.148 \\
Blank-plasma B & 37.258 & 35.773 & 34.800 & 37.677 & 39.142 & 39.848 & 37.417 & 5.145 \\
Blank-plasma C & 36.947 & 35.467 & 34.461 & 37.542 & 39.805 & 40.577 & 37.466 & 6.347 \\
Blank-plasma D & 36.184 & 34.714 & 33.716 & 39.520 & 40.702 & 41.564 & 37.733 \\
Blank-plasma E & 35.734 & 34.270 & 33.278 & 37.375 & 39.517 & 40.260 & 36.737 \\
Blank-plasma F & 34.415 & 32.970 & 31.945 & 36.716 & 36.436 & 36.872 & 34.892 \\
\hline
\end{tabular}

$\mathrm{RSD}$, relative standard deviation. 